\title{
EchoGéo
}

$58 \mid 2021$

Varia

\section{Pratiques et contextes}

Éditorial

Serge Weber

\section{CpenEdition}

Journals

Édition électronique

URL : https://journals.openedition.org/echogeo/22744

DOI : $10.4000 /$ echogeo.22744

ISSN : 1963-1197

Éditeur

Pôle de recherche pour l'organisation et la diffusion de l'information géographique (CNRS UMR 8586)

Référence électronique

Serge Weber, «Pratiques et contextes », EchoGéo [En ligne], 58 | 2021, mis en ligne le 31 décembre 2021, consulté le 12 février 2022. URL : http://journals.openedition.org/echogeo/22744; DOI : https:// doi.org/10.4000/echogeo.22744

Ce document a été généré automatiquement le 12 février 2022.

EchoGéo est mis à disposition selon les termes de la licence Creative Commons Attribution - Pas d'Utilisation Commerciale - Pas de Modification 4.0 International (CC BY-NC-ND) 


\title{
Pratiques et contextes
}

\author{
Éditorial
}

\section{Serge Weber}

1 Ce numéro Varia propose des escales dans plusieurs grandes ou très grandes agglomérations, de Niamey à l'île-de-France, en passant par Dubaï et par le corridor ouest-africain, et s'attarde dans des espaces moins denses au Burkina Faso et au Sénégal. Chacun des textes détecte une ou plusieurs manières dont les pratiques des acteurs sociaux ou des habitants interrogent les effets de contexte à plusieurs échelles. C'est par l'Afrique de l'Ouest que s'ouvre la rubrique Sur le champ.

2 Adam Abdou Alou explore les facteurs d'influence des choix résidentiels en zone inondable à Niamey. Comme dans beaucoup d'autres villes, la pauvreté de nombreux ménages modestes, conjuguée à la croissance urbaine et aux difficultés d'accès au logement, s'accompagne d'une part d'informalité dans l'extension urbaine qui pèse sur la prévention des risques. Dans un contexte de sécheresse accrue depuis les années 1970, une longue période sans crue a donné lieu à une extension de l'habitat informel dans les zones inondables proches du fleuve Niger, en particulier dans le cinquième arrondissement de la capitale. L'auteur cherche à comprendre les facteurs ayant conduit des ménages relogés après la crue catastrophique de 2012 à revenir dans cette zone, où ils ont été à nouveau victimes des crues de 2013 puis de 2020. Il explore donc les dimensions sociales et économiques de la mobilité résidentielle, éclairant ainsi la thématique de la mémoire des risques au gré d'une situation bien particulière.

3 Le deuxième article dévoile les difficultés de contenir l'informalité dans l'extraction artisanale de l'or, au sud-ouest du Burkina Faso, comme le recommandent les institutions internationales. Il explore les jeux complexes d'acteurs impliqués dans la filière, les verrous juridiques et sociaux ainsi que les enjeux environnementaux sousjacents dans la normalisation d'une pratique d'orpaillage dont la régulation n'est pas aisée. L'article cherche donc à comprendre pourquoi, dans un contexte d'attractivité mondiale de l'or, l'amélioration des techniques d'exploitation n'a pas favorisé la formalisation attendue. Cet article a été rédigé par deux chercheurs du Burkina Faso, Edith Sawadogo et Dapola Evariste Constant $\mathrm{Da}^{\dagger}$. Figure centrale de la géographie à 
l'université de Ouagadougou, Dapola Evariste Constant Da est décédé après l'écriture de ce texte ; sa publication est une facon de lui rendre hommage.

El hadj Bara Deme et Moustapha Deme proposent une réflexion sur les effets de l'évolution de la pêche artisanale sur les sociétés du littoral sénégalais. La pêche des petits pélagiques est essentielle à la sécurité alimentaire (le poisson représentant $75 \%$ des protéines animales consommées au Sénégal) et assure localement un emploi direct de transformation (fumage), surtout féminin. Mais la croissance rapide de la pêche artisanale, permise par l'abondance de la ressource dans cette zone d'upwelling, doit aussi répondre au développement de la filière d'exportation et à la hausse de la demande des usages non alimentaires (farines et huiles). Cette évolution a entraîné la division par deux de la consommation intérieure de ces poissons et menace la durabilité de la ressource, mettant ainsi en péril des équilibres fragiles.

Rebecca Cadeau étudie la manière dont les filiations associatives récentes contribuent à reconfigurer «un tout petit monde » qui n'est pas si petit que cela : la communauté haïtienne d'île-de-France, fortement structurée par un tissu associatif très dense. Pour ce faire, elle dresse un panorama historique et géographique des différentes territorialités diasporiques et se livre à une ethnographie des réseaux socionumériques mobilisés par ces associations. Cette recherche, s'inscrivant dans le sillage de recherches pionnières qui ont montré comment les «migrants connectés » déjouent les a priori sur le déracinement, décrit la manière dont les utilisateurs de ces réseaux articulent distance et échelles, reconfigurent les territorialités et parviennent à « faire pays » en se faisant les "artisans » du lien entre la société haïtienne et le reste du monde.

6 Du nouveau sur Dubaï, c'est ce qu'apporte l'article de Raphaël Le Magoariec en clôture de ce dossier, en apportant de l'inédit sur un aspect très peu étudié jusqu'ici. Il choisit trois grands types de lieux et de sphères d'activités sportives pour explorer les contrastes d'une société citadine qui ne laisse pas de surprendre. Les clubs professionnels interrogent à nouveaux frais la question récurrente des contours de la nationalité émiratie : confrontés à une compétition mondiale mais à un vivier restreint, ils fonctionnent comme un des rares points où la frontière de l'accès à la nationalité pour les étrangers peut être poreuse. Les activités sportives des travailleurs qualifiés et de leurs familles sont canalisées par la floraison d'une série de structures privées qui reflètent les standards d'un cosmopolitisme mondialisé où performance physique et bien-être font pendant à un système migratoire utilitariste et sélectif. Les activités amateur des travailleurs subalternes, dont beaucoup sont originaires du sous-continent indien, inventent des usages informels des interstices urbains et sont l'occasion d'un ressourcement diasporique où la passion pour certains sports collectifs comme le cricket joue comme un dénominateur commun. Stratifiée certes, à l'image de cette société kaléidoscopique, l'omniprésence de l'activité sportive pourrait faire office de mode de régulation à la fois sociale et idéologique.

Les dynamiques urbaines sont le point commun des trois rubriques de ce numéro.

8 La rubrique Sur le métier donne la parole à une enseignante-chercheuse en urbanisme et aménagement, Elsa Vivant, qui s'interroge et publie depuis longtemps sur le métier même d'urbaniste. Son dernier livre, L'impasse. Scènes de l'urbanisme ordinaire, repousse les limites de l'écriture scientifique. Elle a développé, durant des années d'investigation, une attention renforcée aux diverses professions des diplômées de cette spécialité, que ce soit auprès des jeunes générations d'experts ou sur le terrain, en 
observant, écoutant et décryptant les positions, les prises de parole et les non-dits des différentes catégories d'acteurs impliqués dans des opérations de rénovation urbaine. Cette expérience multiforme, elle a choisi d'en proposer une écriture radicalement différente: seule une fiction à la fois documentaire et poétique pouvait restituer l'effervescence et l'ambivalence des rapports de travail dans le quotidien de l'opération urbaine.

9 La fabrique de la ville fait également, au sens propre, l'objet de la rubrique Sur l'écrit, autour du livre d'Armelle Choplin, Matière grise de l'urbain. La vie du ciment en Afrique. Son livre propose lui aussi une originalité éditoriale : il est disponible en ligne en plus de la version imprimée et offre une série de ressources documentaires en accès libre. Suivre les sacs de ciments a amené l'autrice à parcourir l'immense corridor urbain ouest-africain, qui longe le littoral en traversant les frontières, depuis Accra jusqu'à Lagos. Elle a rencontré diverses figures d'entrepreneurs, depuis le revendeur informel de sacs de ciment jusqu'aux grandes figures de "l'Africapitalisme » qui, concurrençant depuis peu les géants mondiaux du béton, contribuent à "africaniser un matériau colonial ». Autant la "vie sociale du ciment » révèle les changements sociaux et en particulier le rôle croissant des femmes dans la construction, autant elle a de lourdes conséquences environnementales, tant dans l'extraction du sable que dans ses effets polluants, qui commencent à alerter les consciences dans ce corridor où s'invente un nouveau modèle urbain.

Morgane Flégeau relate, pour la rubrique Sur l'image, la manière dont elle a utilisé la photographie dans sa recherche sur les dynamiques paysagères des marges franciliennes affectées par l'extension périurbaine de la métropole parisienne. Le dispositif de collecte qu'elle a construit est original: le long de plusieurs transects radiaux parcourus à pied à travers la Ceinture verte d'Île-de-France, elle a pris des clichés tous les kilomètres, parfois contrainte à des détours ou à des supplétifs lorsqu'elle rencontrait des obstacles infranchissables. Ces clichés, formant une base de données photographique, lui permettent de mieux qualifier les arrangements paysagers, plus ou moins urbanisés, plus ou moins privatisés, ainsi que les ruptures paysagères telles qu'elle les a vécues en marchant, remettant en question l'idée d'un gradient d'urbanité décroissant du centre vers la périphérie. Ses réflexions viennent combler un manque dans l'articulation des échelles pour apprécier les effets paysagers très localisés d'aménagements urbains qui se déclinent à une échelle métropolitaine. 